\title{
TCAD-based DLTS simulation for analysis of extended defects
}

\author{
Artur Scheinemann* and Andreas Schenk \\ ETH Zurich, Gloriastr. 35, 8092 Zurich, Switzerland
}

Received 20 June 2013, revised 24 July 2013, accepted 31 October 2013

Published online 19 December 2013

Keywords deep level transient spectroscopy (DLTS), dislocation loops, extended defects, leakage currents, technology computer aided design (TCAD)

* Corresponding author: e-mail scheinemann@iis.ee.ethz.ch, Phone: +41-44-6325957, Fax: +41-44-6321194

The fabrication technology of extra-functionality CMOS devices involves process steps which lead to high damage in the silicon lattice. Amorphizing implants and simultaneous reduction of thermal budgets to gain better control of the formation of ultra-shallow junctions render the presence of extended defects in active regions unavoidable. In particular, dislocation loops (DLs) have proven to be stable under thermal treatment. To better understand the electrical properties of DLs and their impact on the leakage current we developed an analytical tool to extract defect parameters from measured Deep Level Transient Spectroscopy (DLTS) signals and capacitance transients. Commercial process and device simulators are used to test the plausibility of applied defect models and the basic assumptions about the electrical activity of DLs.

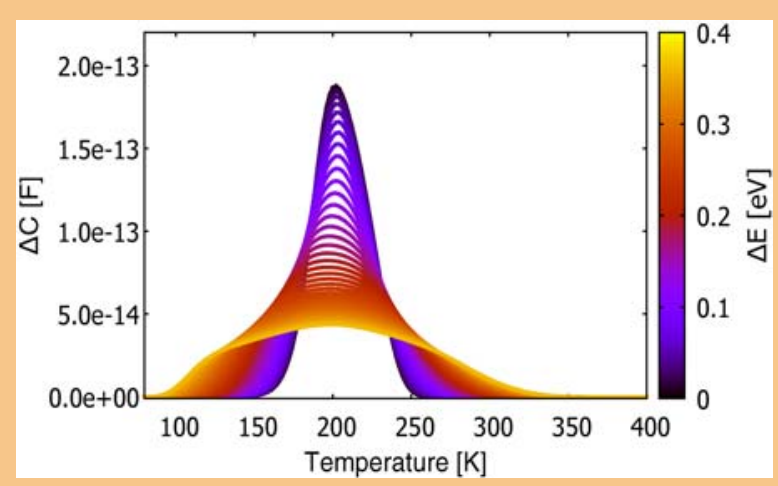

Simulation of DLTS peak broadening caused by the broadening of a defect level distribution in the band gap of silicon.
1 Introduction Extended defects resulting from preamorphization processes and amorphizing implants are frequently reported to be related to increased leakage currents $[1,2]$. Especially dislocation loops (DLs), $\{311\}$ rod-like defects and small interstitial clusters (ICs) are of growing interest. All these defects are evolutionary successors of the interstitial point defect during thermal processing [3]. DLs prove to be thermally very stable [4] and, therefore, a considerable concentration of these defects often remains in the active regions of the final CMOS device.The position of the defects is an important constraint, since only defects in the depletion region can contribute to leakage currents. Besides their unintentional introduction, extended defects have been used in the field of channel stress engineering [5-7]. A better understanding of the mechanisms that govern the leakage caused by extended defects is of crucial importance in order to predict the electrical behavior of the next-generations advanced CMOS devices.

A consistent model of the electrical properties should as well be able to explain the impact that the extended nature of defects has on deep level transient spectroscopy (DLTS) measurements. In this paper we present a new approach to simulate DLTS signals reducing the amount of necessary approximations to a minimum and allowing to test the impact of different physical properties of a defect state on the DLTS characterization. Section 2 will start with a brief discussion of the assumed origin of electrical activity of DLs. Section 3 outlines and compares different approaches to obtain an analytical/simulated DLTS signal. Section 4 analyzes and evaluates the implications of different assumptions on the physical nature of the DLs. Finally, the paper is concluded with a discussion of the results and further investigations that need to be considered. 


\section{Electrical properties of dislocation}

loops Extended defects have been repeatedly in the focus of research. Read Jr., one of the pioneers of the SRH theory, already in 1954 seized on the electrical properties of dislocations [8]. Since these early days the knowledge about extended defects has been greatly improved. This is especially true from the process technical view point [911]. Still when it comes to obtain a clear and conclusive picture of the actual physical process that increases the leakage in the presence of DLs, no model is available to explain or even to predict the characteristics of devices containing DLs.

2.1 Origin of electrical activity As stated in Ref. [12] it can be assumed that the electrical activity caused by dislocations and dislocation loops is due to the presence of impurities that are bound to the extended defects (as schematically shown in Fig. 1) and introduce deep levels into the band gap. In the case of DLs these impurities are supposed to be bound at the periphery of the disc-shaped defect [13]. Hence the concentration of electrically active defects is found to be

$$
N_{\mathrm{T}} \sim \pi \sqrt{C_{\mathrm{DL}} \times D_{\mathrm{DL}}}
$$

where $C_{\mathrm{DL}}$ denotes the concentration of interstitials bound to DLs and $D_{\mathrm{DL}}$ is the concentration of the DLs [3]. Eq. (1) is not a strict equality since the population of the DLs periphery needs to be modeled by an additional parameter which is the ratio between the number of peripheral interstitial sites and the number of impurities bound to the defect. All necessary quantities were obtained by process simulation with Sentaurus-Process (S-Process) [14].

2.2 Coulomb repulsion at extended defects The defect concentration obtained in Eq. (1) is not equivalent to the case of homogeneously distributed point defects with the

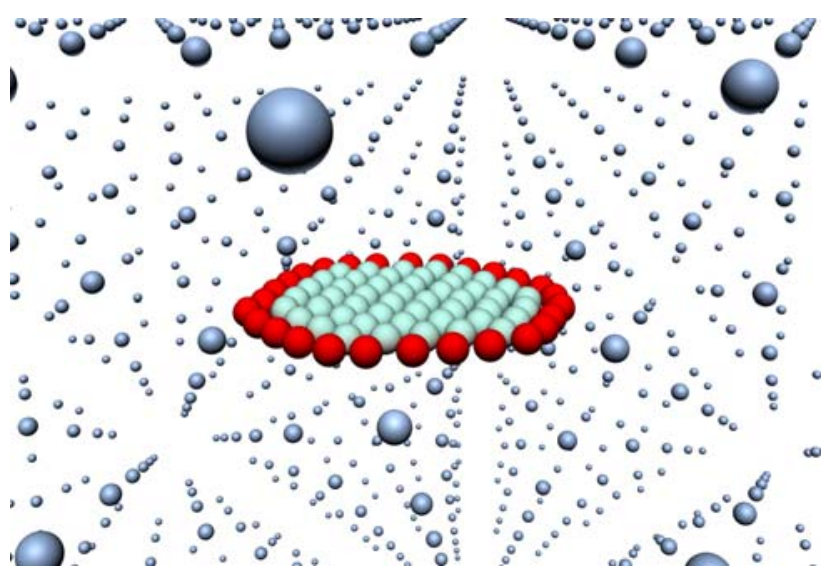

Figure 1 Schematic representation of a dislocation loop defect in the silicon lattice with electrically active periphery. The in-plane distances of the defect constituents are visualized on a different scale to develop a clearer picture of the defect shape. same concentration. While at a concentration of $10^{15} \mathrm{~cm}^{-3}$ for a homogeneous distribution of defects the average distance between two defects is $>60 \mathrm{~nm}$, in the case of defects bound to a DL the distance between defects reduces to $<1 \mathrm{~nm}$. This has significant implications on the defect properties. Read Jr. [8] considered the energy contributions arising from the Coulomb repulsion of charged defects on a dislocation in germanium. By using the circular geometry of Fig. 2a, we find for DLs that the Coulomb energy is given by

$$
E_{\text {Coulomb }}^{\text {even }}=\frac{\mathrm{e}^{2}}{4 \pi \varepsilon \varepsilon_{0}} \frac{1}{2 R}\left\{1+\sum_{i=1}^{\left(n_{\mathrm{T}}-2\right) / 2} \frac{2}{\cos \left(i \pi / n_{\mathrm{T}}\right)}\right\},
$$

$$
E_{\text {Coulomb }}^{\text {odd }}=\frac{\mathrm{e}^{2}}{4 \pi \varepsilon \varepsilon_{0}} \frac{1}{2 R} \sum_{i=1}^{\left(n_{\mathrm{T}}-1\right) / 2} \frac{2}{\sin \left(i \pi / n_{\mathrm{T}}\right)},
$$

where $n_{\mathrm{T}}$ is the number of charged defect sites (either odd or even). Both expressions give the same value for big enough DLs. The derivation of Eqs. (2) and (3) is based on the assumption that captured carriers can freely migrate along the periphery of the DL. Therefore, on capture and emission of one carrier the ensemble will redistribute immediately to homogeneously maximize the mutual distances. Since this assumption is hardly justified (as will be discussed in Section 5) a comparison was made with the opposite case of completely localized carriers bound to the site where they are actually captured. This has been accomplished numerically starting with a fully charged DL: The first site to emit its carrier is selected randomly. Successively the new Coulomb energy of every left carrier is computed and the carrier with the highest energy is emitted next. This procedure is repeated until the DL is completely empty.

The difference between the two cases is shown in Fig. 2b. As this difference is rather small but, on the other hand, the case of localized carriers results in a high computational burden, the analytical expressions (2) and (3) for the Coulomb repulsion energy were used instead.

3 DLTS simulation The deep level transient spectroscopy (DLTS) as pioneered by Lang [15] has advanced to a versatile and easily applicable characterization technique. A rich pool of literature on the DLTS technique is available [15-17]. Although there are improvements and evolutions of the original idea, the simple box-car approach remains perfectly suited for simulations due to its simplicity and the large amount of available data in publications.

3.1 Analytical solution The DLTS technique measures the transient relaxation of a defect system to a new stationary state after it has been pulled out of its original stationary state by the application of a voltage pulse $V_{r, p}$. The measured capacitance transient can be directly related to a transient of the number of occupied defect sites $n_{\mathrm{T}}$ or the occupation probability $f=\left(n_{\mathrm{T}} / N_{\mathrm{T}}\right)$. Since changes in the occupation of a defect are induced by the capture $\left(c_{\mathrm{n}}, c_{\mathrm{p}}\right)$ and 


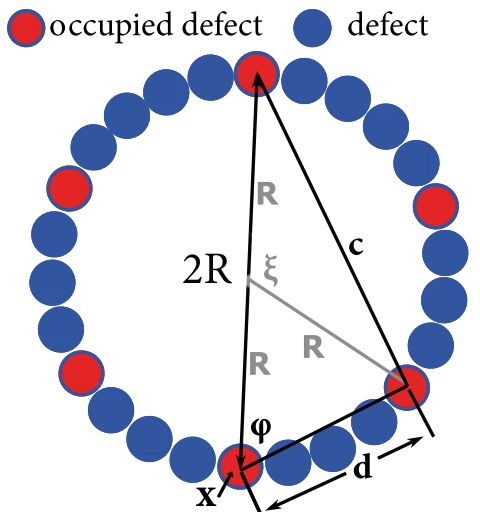

a) Loop Geometry

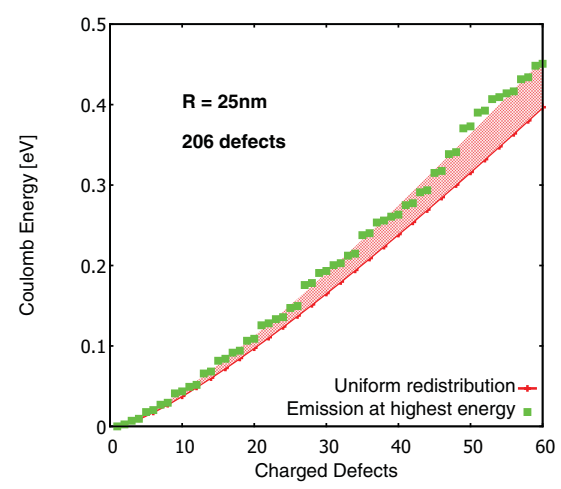

b) Coulomb Energy

Figure 2 (a) Schematic view of DL periphery partially occupied by captured carriers with geometry used to derive the Coulomb contribution to the defect level. (b) Deviations between the assumption that captured carriers can redistribute instantaneously along the dislocation loop (solid line) and the case where they are bound to their site while capture and emission probabilities vary along the periphery of the defect with the local Coulomb energy contribution (square symbols). The shaded area indicates $15 \%$ difference from the original analytical expression.

emission $\left(e_{\mathrm{n}}, e_{\mathrm{p}}\right)$ of both carrier types, a differential equation for the occupation of the defects

$$
\begin{aligned}
\frac{\mathrm{d} n(t, T)}{\mathrm{d} t}= & -n(t, T)\left[e_{\mathrm{n}}(T)+c_{\mathrm{p}}(T)\right] \\
& +\left(N_{\mathrm{T}}-n(t, T)\right)\left[e_{\mathrm{p}}(T)+c_{\mathrm{n}}(T)\right]
\end{aligned}
$$

has to be solved. All quantities generally depend on the temperature $T$. To obtain an explicit solution for electron emission after application of a release pulse, the capture rates $c_{\mathrm{n}}, c_{\mathrm{p}}$, and the hole emission rate $e_{\mathrm{p}}$ are usually omitted. In the further analytical discussion we will consider defect levels in the upper half of the band gap which justifies these assumptions. Then the solution of Eq. (4) is

$$
n(t, T)=n\left(t_{0}, T\right) \mathrm{e}^{-t e_{\mathrm{n}}(T)}
$$

with

$$
e_{\mathrm{n}}=v_{\mathrm{th}, \mathrm{n}} \sigma_{\mathrm{n}} N_{\mathrm{C}} \mathrm{e}^{-\left(\left(E_{\mathrm{C}}-E_{\mathrm{T}}\right) /\left(k_{\mathrm{B}} T\right)\right)} .
$$

The DLTS signal as function of temperature $T$ is constructed from the difference of the occupation numbers at two different sampling times $t_{1}$ and $t_{2}$ :

$$
S\left(T, t_{1}, t_{2}\right)=n\left(t_{1}, T\right)-n\left(t_{2}, T\right) .
$$

Reasonable agreement between measured DLTS peaks and analytical theory is only found for point defects. Unfortunately, Eq. (5) is a poor description of the reality when it is compared to DLTS measurements of extended defects, as can be seen in Fig. 3. The origin of the small midgap peak that is observed in the measurements and shown in Figs. 3 and 4 is not yet clarified. In Ref. [19] very similar mid-gap levels with a high concentration were found in samples with ICs and $\{311\}$ defects. In such samples the responsible defect is reported to anneal out at temperatures of
700 and $950{ }^{\circ} \mathrm{C}$, respectively. The sample discussed in this paper which containes DLs was annealed at $1020^{\circ} \mathrm{C}$. Therefore, the small mid-gap peak cannot be related to ICs or $\{311\}$ defects.

The broadened DLTS signal caused by a defect at $E_{\mathrm{T}} \approx E_{\mathrm{C}}-0.35 \mathrm{eV}$ is similar to the DL-related E3 level reported by Ayres et al. [20]. They found an activation energy of $0.38 \mathrm{eV}$ and a standard deviation of the assumed Gaussian broadening of $27 \mathrm{meV}$. Another similarity exists with the famous dislocation-related "C-line" extensively discussed e.g., in Ref. [12] with reported activation energy of $E_{\mathrm{T}} \approx E_{\mathrm{C}}-(0.37-0.43) \mathrm{eV}$ and line broadening of $(10-50) \mathrm{meV}$. Interestingly, those broadenings are much smaller than the value necessary to reproduce the strong broadening of the main peak in Figs. 3 and 4.

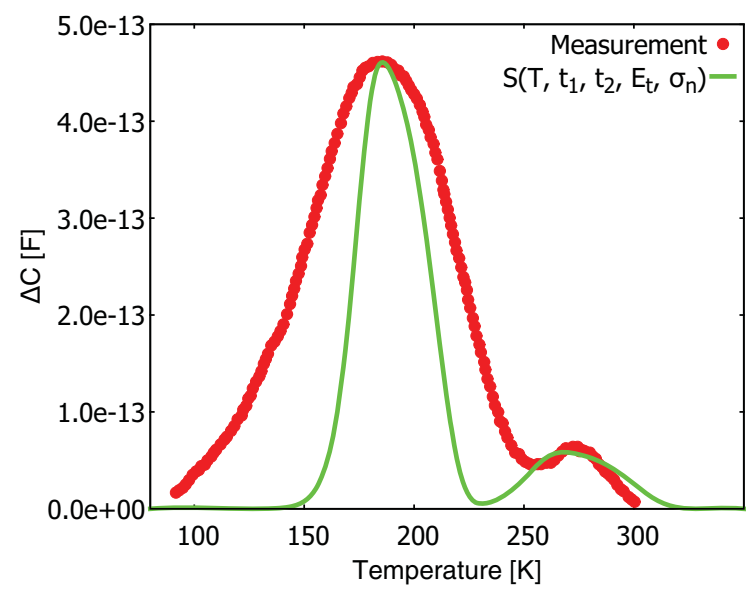

Figure 3 Analytical solution from Eq. (7) plotted together with a measured DLTS peak at $E_{\mathrm{T}, 1} \approx E_{\mathrm{C}}-0.35 \mathrm{eV}$ which is due to DLs [18]. The second peak is found to be at $E_{\mathrm{T}, 2} \approx E_{\mathrm{C}}-0.54 \mathrm{eV}$. For the analytical solution the measured energy levels and $\sigma_{\mathrm{n}, 1}=\sigma_{\mathrm{n}, 2}=10^{-15} \mathrm{~cm}^{2}$ were used as parameters. 


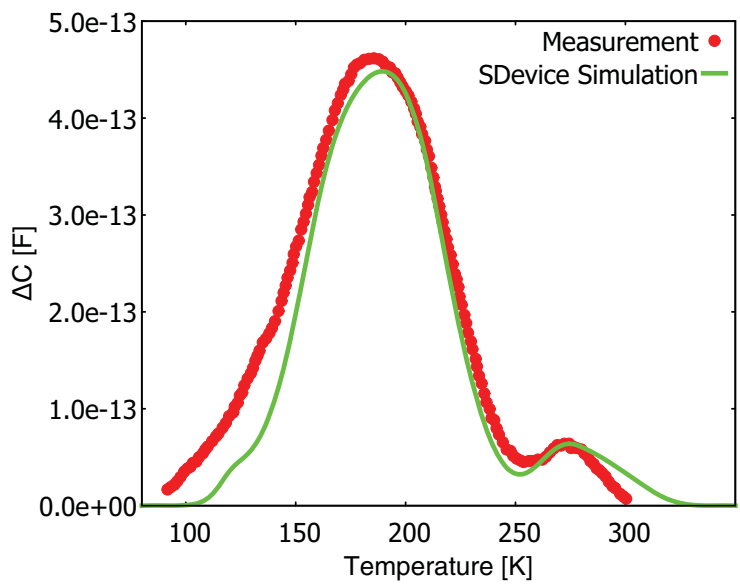

Figure 4 "Best-fit" simulation of the measured DLTS signal of Fig. 3 with parameters of main peak: $E_{\mathrm{T}, 1}=E_{\mathrm{C}}-0.33 \mathrm{eV}, \sigma_{\mathrm{n}, 1}=$ $1 \times 10^{-15} \mathrm{~cm}^{2}$, and assuming 30 uncoupled levels distributed over an energy interval $\Delta E=0.2 \mathrm{eV}$ around $E_{\mathrm{T}, 1}$. Parameters of the small mid-gap peak are: $E_{\mathrm{T}, 2}=E_{\mathrm{C}}-0.55 \mathrm{eV}, \sigma_{\mathrm{n}, 2}=\sigma_{\mathrm{p}, 2}=1 \times 10^{-15} \mathrm{~cm}^{2}$.

3.2 Device simulation of the DLTS signal Besides the omission of $c_{\mathrm{n}}, c_{\mathrm{p}}$, and $e_{\mathrm{p}}$, in most applications it is silently assumed that

(1) $N_{\mathrm{T}} \neq N_{\mathrm{T}}(\boldsymbol{x})$ i.e., defects are homogeneously distributed throughout the device;

(2) $n\left(t_{0}, T\right)=N_{\mathrm{T}}$; i.e., defects are fully occupied at $t=0$.

All of these approximations can be avoided when the DLTS signal is obtained from a self-consistent device simulation. This is easily done by a transient simulation with the same voltage pulse sequence that is used in the experiment. In our case Sentaurus-Device (SDevice) [21] was used. A script is then applied to extract the necessary information from the simulation results, which is the capacitance and voltage at the predetermined time points, and the DLTS signal is constructed from them. Such simulations can be used to validate assumptions on the properties of a given defect species, such as the defect density profile $N_{\mathrm{T}}(\boldsymbol{x})$, the defect level $E_{\mathrm{T}}$, and the capture cross sections $\sigma_{\mathrm{n}}, \sigma_{\mathrm{p}}$. Figure 4 shows the simulation of a DLTS measurement of a Schottky diode containing DLs as a result of high-dose implantation with Ge [18]. The key steps of the fabrication process for this sample are:

(1) n-type wafer with substrate doping concentration of $10^{15} \mathrm{~cm}^{-3}$

(2) Ge implant with $150 \mathrm{keV}$ and a fluence of $3 \times 10^{14} \mathrm{~cm}^{-2}$,

(3) Annealing at $1000^{\circ} \mathrm{C}$ for $15 \mathrm{~min}$,

(4) Additional anneal at $1020^{\circ} \mathrm{C}$ for $120 \mathrm{~min}$ to reduce leakage.

The defect concentrations $N_{\mathrm{T}, i}$ remain fitting parameters in both approaches (analytical solution, Fig. 3 and device simulation, Fig. 4). Although the process simulation yields the defect profiles according to the description in Section 2, the DLs might be only partially decorated with point-like impurities.

The dependence of the peak position on $E_{\mathrm{T}}$, which can also be derived from the analytical solution and which is up to first order linear

$$
T_{\max } \sim \frac{E_{\mathrm{C}}-E_{\mathrm{T}}}{k_{\mathrm{B}}},
$$

is well reproduced (see Fig. 5). Furthermore, a dependence of the amplitude on $E_{\mathrm{T}}$ is observed although the analytical theory predicts that the amplitude only depends on the two sampling times [15]. Most likely this is caused by the temperature dependence of $n\left(t_{0}\right)=n(t=0)$, i.e., the reducing steady-state trap occupation at increased temperatures. Furthermore, the device simulation not only renders all simplifications unnecessary, it also allows to perform DLTS simulations where a numerical solution looses its validity due to conditions that violate the simplifying assumptions. This is especially the case, when the defect concentration is comparable or even higher than the concentration of shallow dopants.

Although this approach has its advantages over the analytical solution of the DLTS signal, a high level of parallelization leads to a high demand in licenses of the used software, since every temperature point is simulated separately. Furthermore, the device simulation generates a lot of overhead, which is not necessarily needed for the actual DLTS simulation leading to a significant waste of CPU time. Another disadvantage is the limited possibility to implement new physical models which go beyond the scope of the provided interfaces for model implementation.

3.3 Mixed approach In order to combine the advantage of using the self-consistent electrostatics obtained by S-Device (electric field, free carrier densities, and any

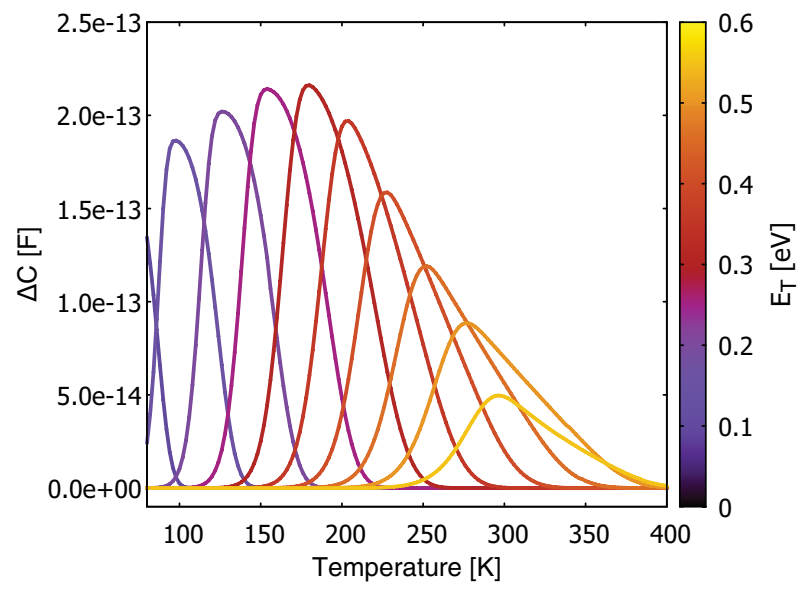

Figure 5. A shift of the defect level position in the band gap from mid-gap to $E_{\mathrm{C}}$ shifts the DLTS peak to smaller temperatures confirming the analytical result that $T_{\max , i} \sim E_{\mathrm{C}}-E_{\mathrm{T}, i}$. 
other quantity of interest) and the defect distributions from process simulation, a modified approach to obtain the DLTS signal was introduced.

(1) Process simulations and stationary device simulations at all temperatures and bias conditions are performed and stored in the initial phase.

(2) Using the pre-computed electrostatics the stationary state occupations of the defects can be computed for the loading pulse $V_{\mathrm{p}}$.

(3) Replacing the electrostatics of the device at the loading pulse $V_{\mathrm{p}}$ with the one at the release pulse $V_{\mathrm{r}}$ and numerically integrating the differential Eq. (4) without any simplifications yields the transient solution of the defect occupation and after extraction of the experimentally determined time points generates the DLTS signal.

This approach is computationally very efficient and gives full flexibility to modify the physical nature of the carrier exchange processes $\left(c_{\mathrm{n}}, c_{\mathrm{p}}, e_{\mathrm{n}}, e_{\mathrm{p}}\right)$.

4 Impact on DLTS signal Applying the previously introduced methodology it is now possible to efficiently test different assumptions about the nature of the underlying defects.

4.1 Coupling of defect levels Due to the spatial proximity of the bound impurities to the dislocation loop interactions between the defects should not be neglected. Such a coupling of defect levels can have significant impact on the electrical properties as shown in Ref. [22]. The coupling of two distinct levels modeled by an inter-level rate was simulated and the coupling strength was varied in both asymptotic directions, from negligible coupling up to very strong coupling. The results are shown in Fig. 6. High coupling strength leads to the absorption of the DLTS peak which is normally seen at higher temperatures and a

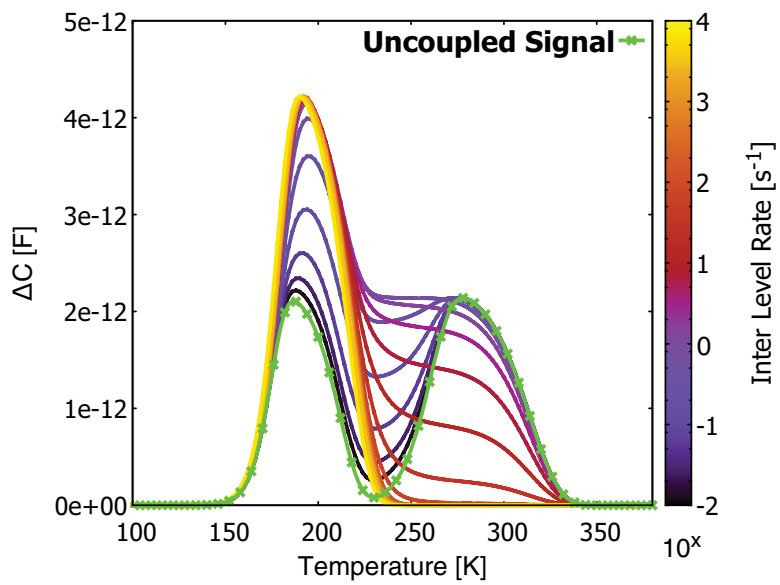

Figure 6. DLTS simulation varying the strength of coupling between two defect levels. Parameters: $E_{\mathrm{T}, 1}=E_{\mathrm{C}}-0.35 \mathrm{eV}$, $E_{\mathrm{T}, 2}=E_{\mathrm{C}}-0.55 \mathrm{eV}, N_{\mathrm{T}, 1}=10^{12} \mathrm{~cm}^{-3}, \quad N_{\mathrm{T}, 2}=1.5 \times 10^{12} \mathrm{~cm}^{-3}$, $\sigma_{\mathrm{n}, i}=\sigma_{\mathrm{p}, i}=10^{-15} \mathrm{~cm}^{2}$.

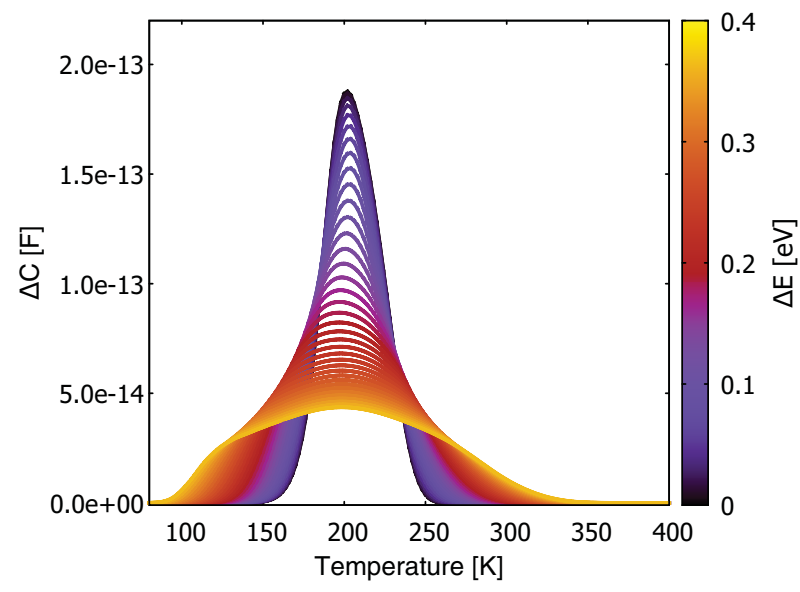

Figure 7. DLTS simulation varying the energy interval $\Delta E$ around $E_{\mathrm{T}}$ using 10 uncoupled levels. Parameters: $E_{\mathrm{T}}=E_{\mathrm{C}}-0.35 \mathrm{eV}$, $N_{\mathrm{T}, i}=10^{12} \mathrm{~cm}^{-3}, \sigma_{\mathrm{n}, i}=\sigma_{\mathrm{p}, i}=10^{-15} \mathrm{~cm}^{2}$.

broadening of the absorbing peak with simultaneous increase of its amplitude is observed. As it is exemplary shown on the abstract picture and can be seen in more detail in Fig. 7 the broadening of a defect level distribution in the band gap causes a broadening of the measured DLTS signal. This situation can be modeled with the analytical expression (5) by a simple superposition of the solutions for different energies with different weighting [19]

$$
\begin{gathered}
\int_{\Delta E} S\left(T, t_{1}, t_{2}, E, \sigma_{\mathrm{n}}\right) \times W(E) \mathrm{d} E \\
=\hat{S}\left(T, t_{1}, t_{2}, E_{t}, \Delta E, \sigma_{\mathrm{n}}\right) .
\end{gathered}
$$

The effects of an increasing coupling strength of such a level distribution can be seen in Fig. 8 where 10 coupled levels were taken into account. Results are similar to the case of only two levels. The high-temperature tail of the peak is being absorbed by the low temperature tail which at the same

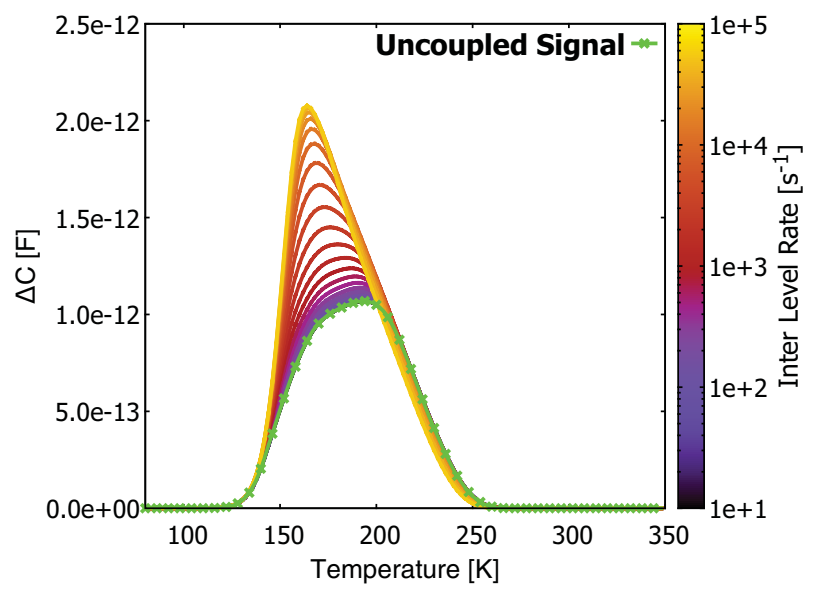

Figure 8. DLTS simulation varying the inter-level rate using 10 coupled levels distributed over an energy interval $\Delta E=0.2 \mathrm{eV}$ around $E_{\mathrm{T}}$. Parameters: $E_{\mathrm{T}}=E_{\mathrm{C}}-0.35 \mathrm{eV}, N_{\mathrm{T}, i}=10^{12} \mathrm{~cm}^{-3}$, $\sigma_{\mathrm{n}, i}=\sigma_{\mathrm{p}, i}=10^{-15} \mathrm{~cm}^{2}$. 
time increases in amplitude. This has the simple explanation that for the high-temperature levels the inter-level rate is faster than the exchange processes with the carrier bands.

The shape of the DLTS peak for a coupled level distribution with its very steep low-temperature edge is not observed in literature. This leads to the conclusion that the coupling of all the levels in the distribution is an unlikely scenario. This conclusion is supported by the multi-phonon theory which allows a strong electron-phonon coupling only for highly localized states, where in turn the strong coupling of the levels in the distribution would lead to a delocalized state over the entire periphery of the DL. Seibt et al. [12] also discarded a fast equilibration of the inter-level system in the case of dislocation-related deep levels.

\subsection{Impact of Coulomb energy on DLTS signal}

The incorporation of the Coulomb repulsion energy which was derived in Section 2.2 is not straight-forward since the stationary occupation of the defect system can no longer by simply computed from the expression

$$
f=\frac{c_{\mathrm{n}}(T)}{c_{\mathrm{n}}(T)+e_{\mathrm{n}}(T)}
$$

when the defect energy depends on the occupation. Therefore, the occupation has to be found by using a selfconsistent iteration loop which successively updates Coulomb energy and resulting defect occupation. The outcome of this simulation can be seen in Fig. 9. The average loop radius is predicted by S-Process to be $>300 \mathrm{~nm}$, assuming an interstitial distance of $7.6 \AA$ [23]. This seems to be an overestimation as TEM images of the sample indicate that the biggest loops are $\approx 200 \mathrm{~nm}$ in diameter. With this preliminary outcome of the process simulation the concentration had to be scaled by a factor of 10 to match the hight of the measured peak.

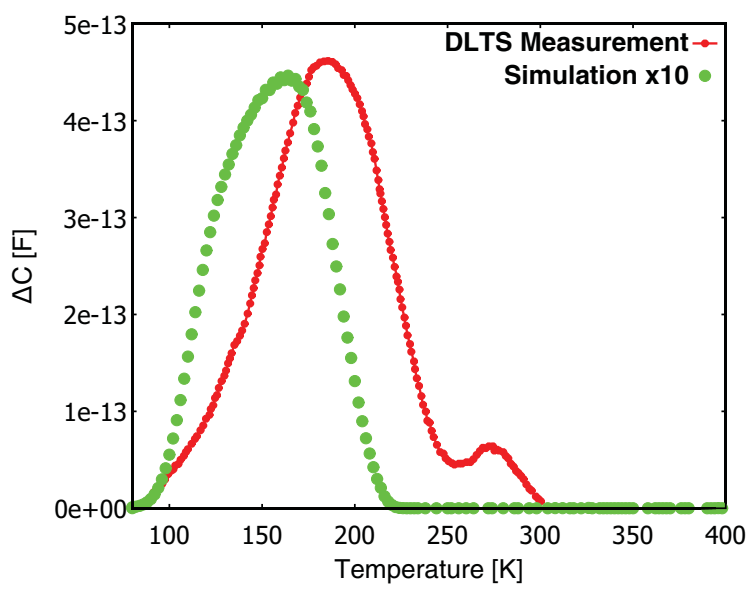

Figure 9 DLTS simulation of DLs taking into account the shifting of the defect level due to Coulomb repulsion. $E_{\mathrm{T}}=E_{\mathrm{C}}-0.35 \mathrm{eV}$, $\sigma_{\mathrm{n}}=\sigma_{\mathrm{p}}=10^{-15} \mathrm{~cm}^{2}$, DL radius and distribution obtained by process simulation.
A significant broadening of the single-level peak is observed. Note that only one defect level $E_{\mathrm{T}}$ was used here (which was assumed to be the same for each defect site on the periphery of the DL). Along with the broadening the amplitude of the DLTS peak is greatly impacted, since due to the Coulomb repulsion only a fraction of the existing peripheral sites can be charged until the Coulomb contribution becomes larger than the distance of the defect level to the Fermi level $E_{\mathrm{F}}$ or to the conduction band edge $E_{\mathrm{C}}$. The "Coulomb ladder" of energy levels results in a shift of the peak to lower temperatures similar to the case of distributed defect levels $E_{\mathrm{T}, \mathrm{i}}$. In order to recover the measured peak position, the defect level has to be increased to $E_{\mathrm{T}, i} \approx E_{\mathrm{C}}-0.4 e \mathrm{~V}$.

5 Conclusions In the present work a new versatile scheme to simulate DLTS signals was presented which allows an efficient but still flexible implementation and evaluation of physical processes that govern the coupling of the defect levels to the respective carrier bands. This can play a key role in understanding the electrical properties of extended defects.

Using the proposed methodology a variety of different assumptions on the defect nature was implemented and tested. The results obtained for the coupling of a multilevel defect are consistent with the multi-phonon theory. The coupling of a mid-gap defect level to a shallower level can suppress the DLTS signal of the mid-gap level. Though it is then not visible in the DLTS characterization it can still contribute to or even dominate the leakage current.

The Coulomb repulsion energy occurring upon the successive charging of a DL was introduced into the DLTS simulation. It was found that the DLTS signal is significantly broadened and shifted towards lower temperatures. The direct usage of device simulation data gives rise to an enormous improvement in the accuracy of the methodology.

Acknowledgements The research leading to these results has received funding from the European Union Seventh Framework Program (FP7/2007-2013) under Grant Agreement No. 258547 (ATEMOX). The invaluable assistance of the following persons is kindly acknowledged: Cloud Nyamhere and François Olivié for the supply with experimental data, Fuccio Cristiano for inspiring discussions and critical remarks and Christoph Zechner, Alexander Tsibizov, and Nikolas Zographos for the process simulations.

\section{References}

[1] K. Ryoo, R. Drosd, and W. Wood, J. Electrochem. Soc. 72, 768 (1989).

[2] R. Duffy, A. Heringa, V. Venezia, M. V. J. Loo, M. Hopstaken, K. van der Tak, M. de Potter, J. Hooker, P. Meunier-Beillard, and R. Delhougne, Solid State Electron. 54, 243 (2010).

[3] N. Zographos, C. Zechner, and I. Avci, Mater. Res. Soc. Symp. Proc. 994 (2007), DOI 10.1557/PROC-0994-F10-01.

[4] S. Libertino and A. L. Magna, Damage formation and evolution in ion-implanted crystalline $\mathrm{Si}$, in: Materials 
Science with Ion Beams, edited by H. Bernas (Springer, Berlin, Heidelberg, 2010), pp. 147-212.

[5] K. Y. Lim, H. Lee, C. Ryu, K. I. Seo, U. Kwon, S. Kim, J. Choi, K. Oh, H. K. Jeon, C. Song, T. O. Kwon, J. Cho, S. Lee, Y. Sohn, H. S. Yoon, J. Park, K. Lee, W. Kim, E. Lee, S. P. Sim, C. G. Koh, S. B. Kang, S. Choi, and C. Chung, Novel stress-memorization-technology (SMT) for high electron mobility enhancement of gate last high- $k /$ metal gate devices, in: Electron Devices Meeting (IEDM), 2010 IEEE International (2010), pp. 10.1.1-10.1.4.

[6] C. Weber, S. Cea, H. Deshpande, O. Golonzka, and M. Liu, Modeling of NMOS performance gains from edge dislocation stress, in: Electron Devices Meeting (IEDM), 2011 IEEE International (2011), pp. 34.4.1-34.4.4.

[7] K. Kuhn, IEEE Trans. Electron Devices 59(7), 1813-1828 (2012).

[8] W. Read, Philos. Mag. 45(367), 775 (1954).

[9] M. Seibt, V. Kveder, W. Schröter, and O. Voß, Phys. Status Solidi A 202(5), 911-920 (2005).

[10] E. Weber, Physica B 340-342, 1-14 (2003).

[11] O. Voß, V. Kveder, and M. Seibt, Phys. Status Solidi A 204(7), 2185-2189 (2007).
[12] M. Seibt, R. Khalil, V. Kveder, and W. Schröter, Appl. Phys. A 96, 235-253 (2009).

[13] V. Moroz and M. Choi, ECS Trans. 33, 221 (2010).

[14] Synopsys Inc. Sentaurus Process User Guide, f-2013.03 edition.

[15] D. Lang, J. Appl. Phys. 45(7), 3023-3032 (1972).

[16] P. Blood and J. Orton, The Electrical Characterization of Semiconductor Majority Carriers and Electron States (Academic Press, New York, 1992).

[17] J. Lauwaert, J. V. Gheluwe, and P. Clauws, Rev. Sci. Instrum. 79, (2008).

[18] Private communication with Cloud Nyamhere and Fuccio Cristiano, LAAS-CNRS, in the EU project ATEMOX.

[19] C. Nyamhere, F. Cristiano, F. Olivie, Z. Essa, and E. BedelPereira, J. Appl. Phys. 113, (2013), DOI 10.1063/1.4804332

[20] J. Ayres and S. Brotherton, J. Appl. Phys. 71, 2702 (1992).

[21] Synopsys Inc. Sentaurus Device User Guide, f-2013.03 edition.

[22] A. Schenk and U. Krumbein, J. Appl. Phys. 78(5), 3185-3192 (1995).

[23] Y. Bar-Yam and J. Joannopoulos, Phys. Rev. B 30(4), 18441852 (1984). 